\title{
THE STABILITY OF THE PLANETARY TRIANGULAR LAGRANGE POINTS
}

\author{
SEPPO MIKKOLA \\ Turku University Observatory, Turku, Finland \\ and \\ K. A. INNANEN \\ Physics Department, York University, Toronto, Canada
}

\begin{abstract}
Numerical, self-consistent, $\mathbf{n}$-body integrations of the solar system show significant indications of medium-term (i.e. several million-year) stability for the various planet-Sun L4,L5 configurations. A progress report of our computations, emphasizing the inner solar system, will be given. There exist interesting possibilities for these locations (including the Earth) as the sites for longer term scientific applications, both pure and applied.
\end{abstract}

\section{Objective}

The observed existence and extensive theoretical analysis of Jupiter's two Trojan asteroid families are our starting points. We ask the question of whether or not some degree of stability at their analogous triangular Lagrange points can exist for the other planets. [From a diagram, it would appear intuitively improbable for such stability to exist.]

\section{Method}

We use a self-consistent $\mathrm{n}$-body computer simulation of the solar system by numerical integration of the equations of motion. In this simulation [emphasizing the situation for the terrestrial planets], we have included all of the planets from Mercury to Saturn. Massless test particles are introduced in the neighborhoods of the classical L4 or L5 points for each terrestrial planet. The system is integrated forward in time, following the time history for each particle in its semi-major axis, eccentricity, inclination and angular separation between particle and planet, as viewed from the Sun.

\section{Results}

For the terrestrial planets, during a 2 million year integration, stability clearly exists. Near the classical L4,L5 points, the test particles oscillate in "tadpole" regions typically of angular size 1.5 degrees. Beyond this, the orbits of the particles remain stable but are horseshoe-like. In Mercury's case, all longer period orbits are likely to be horseshoes. The results and trends for the first million years are shown in Figures 1-4.

Y. Kondo (ed.), Observatories in Earth Orbit and Beyond, 533-536.

(C) 1990 Kluwer Academic Publishers. Printed in The Netherlands. 


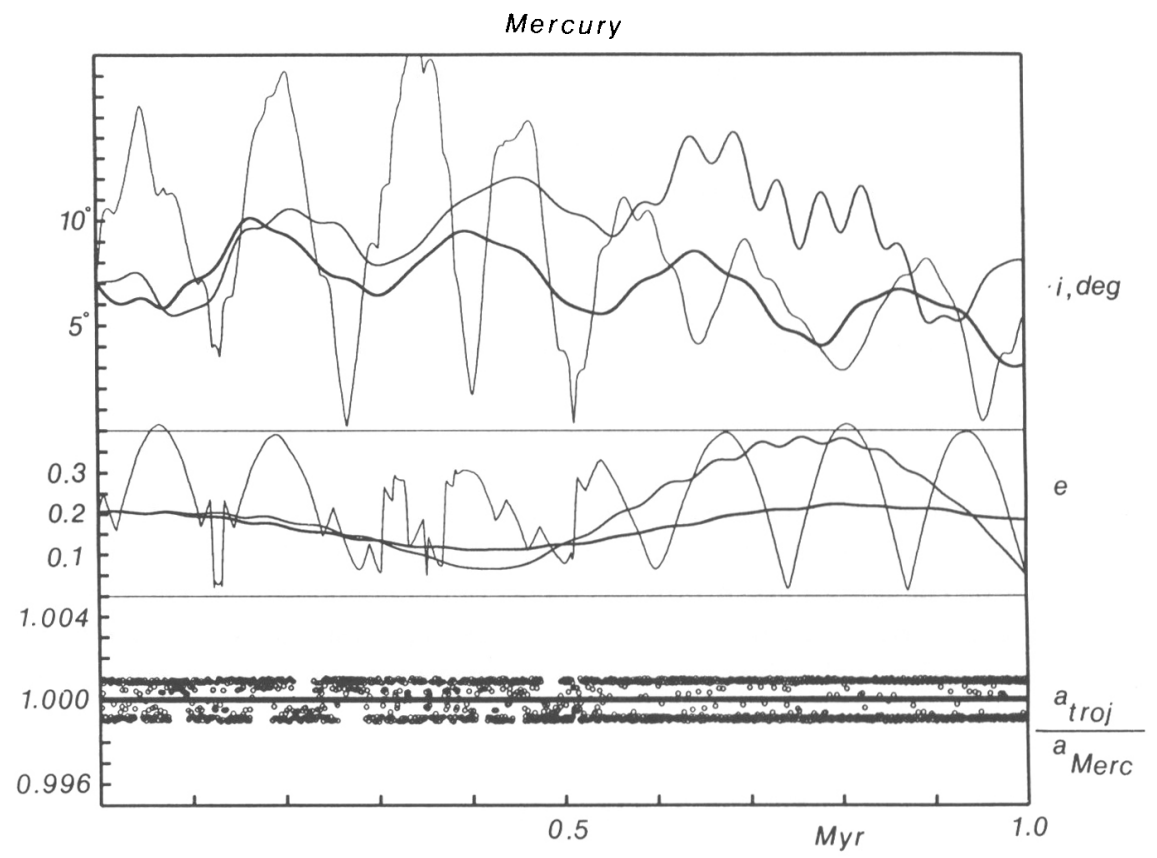

Fig. 1.

VENUS

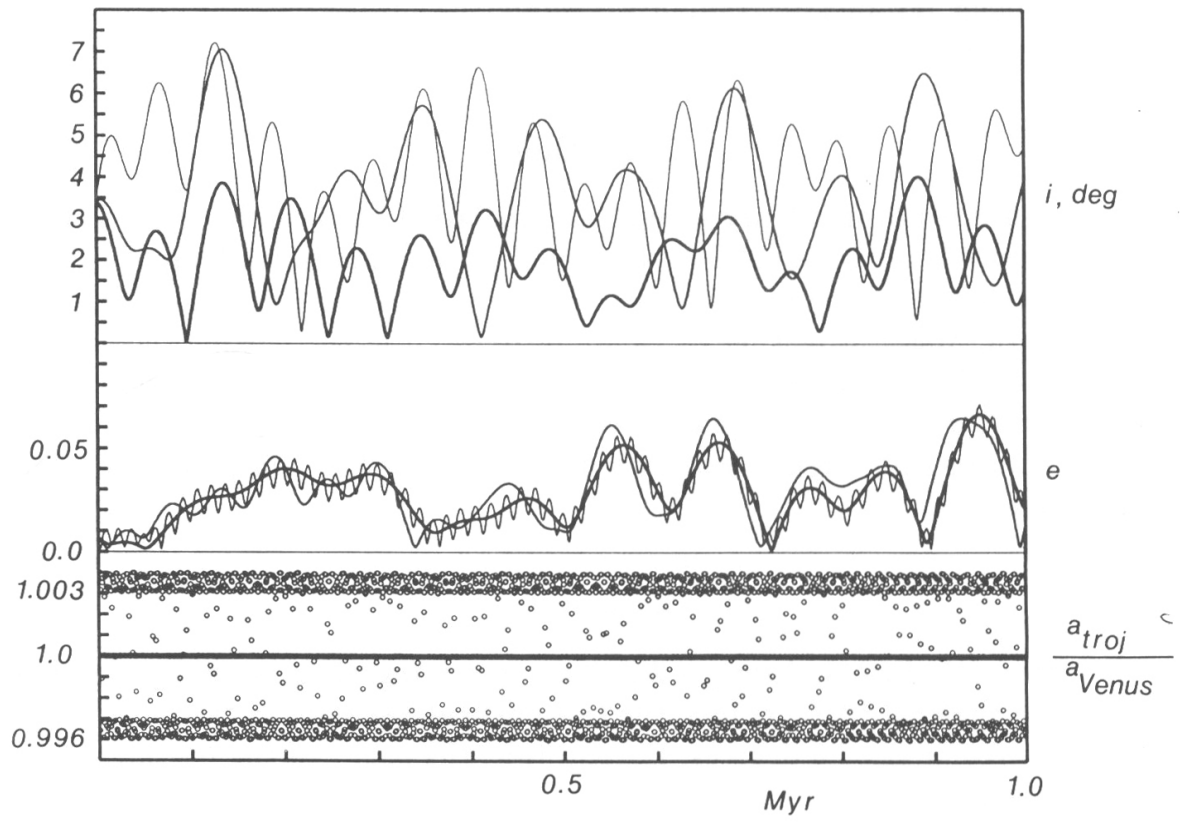

Fig. 2. 
EARTH

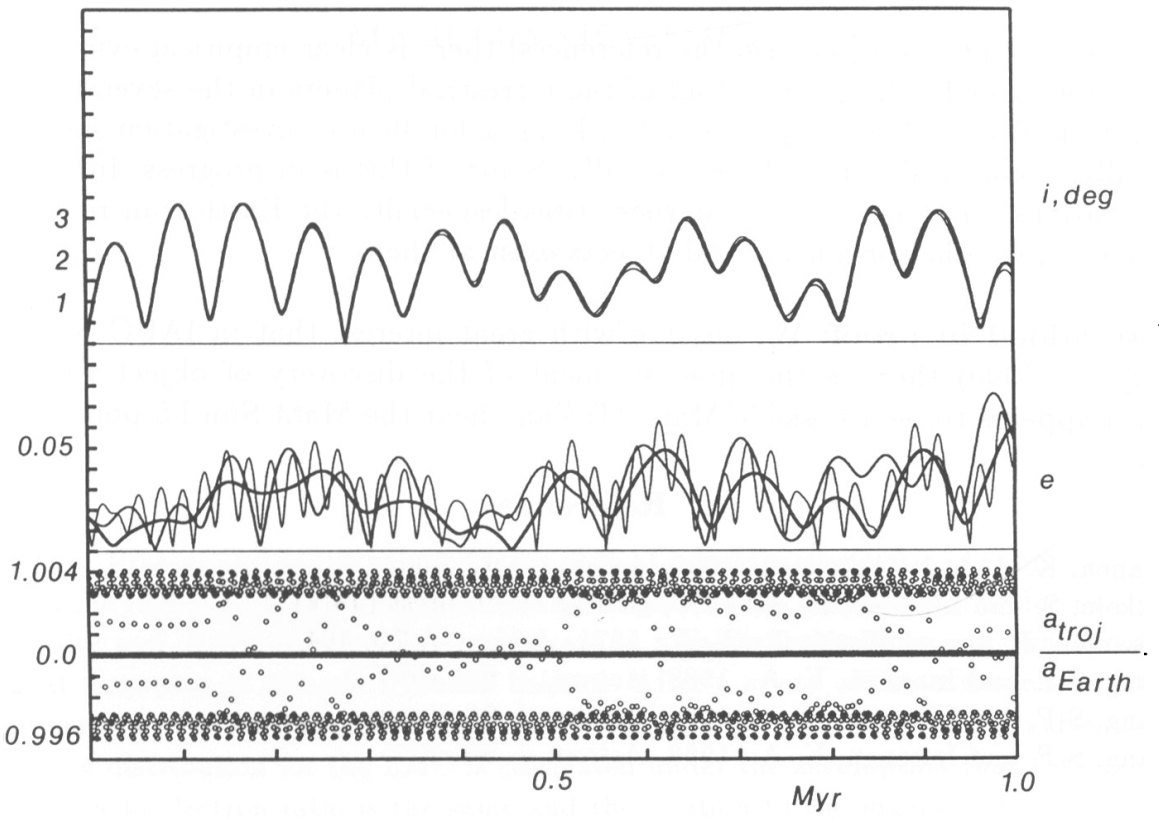

Fig. 3.

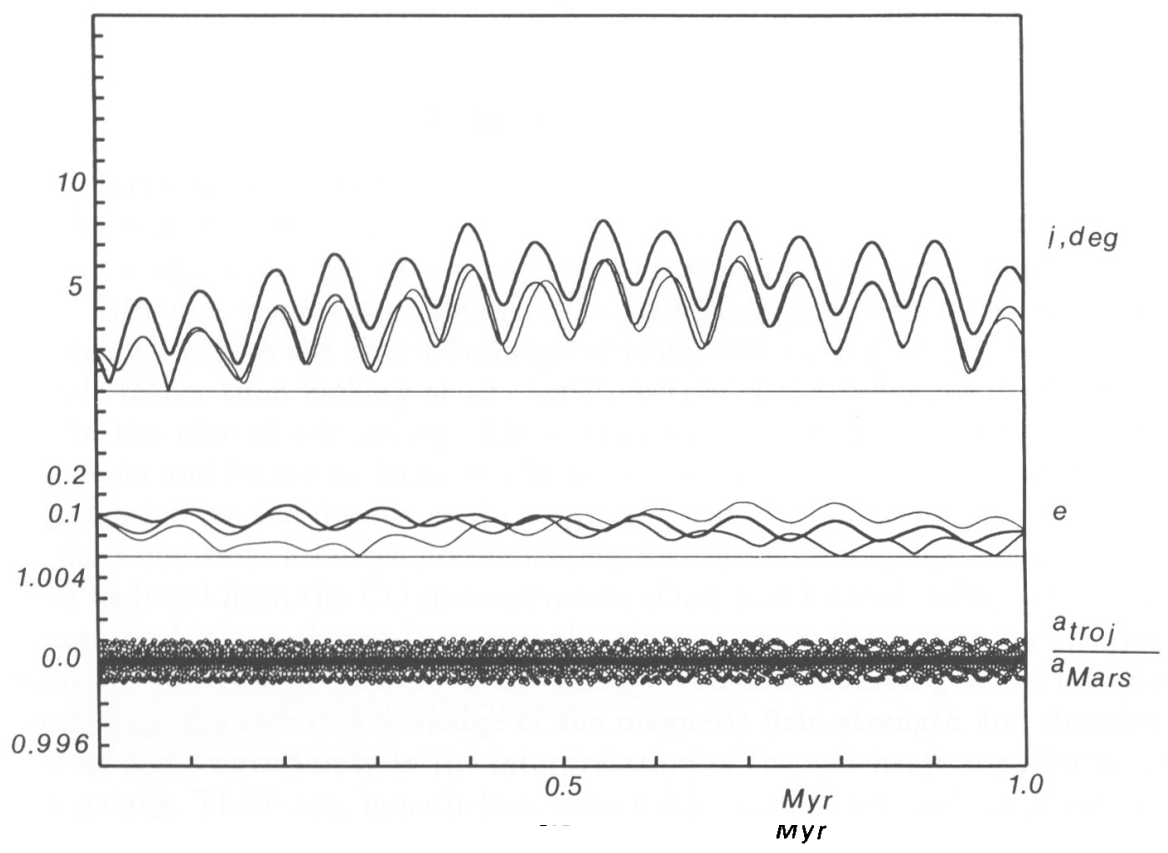

Fig. 4. 


\section{Conclusions}

Contrary to intuition [see also the references] there is clear empirical evidence for the stability of $\mathrm{L}_{4}, \mathrm{~L}_{5}$ points of all of the terrestrial planets in the several million year time frame. The subject is a fertile area for future investigation computationally, theoretically and observationally. Some of this is in progress. Interesting applications for space stations at these sites [especially the Earth] can readily be suggested, whether or not natural objects exist at them.

Note added in proof: We observe with great interest that in IAUC No. 5067 (July 28, 1990) there is the announcement of the discovery of object $1990 \mathrm{MB}$ which appears to be a possible Mars "Trojan" near the Mars-Sun L5 point.

\section{References}

Innanen, K. A. and Mikkola, S.: 1989, Astron. J. 97, 900

Mikkola, S. and Innanen, K. A.: 1990, Astron. J., in press (July)

Weissman, P. R. and Wetherill, G. W.: 1974, Astron. J. 79, 404

Zhang, S-P. and Innanen, K. A.: 1988, Astron. J. 96, 1983

Zhang, S-P. and Innanen, K. A.: 1988, Astron. J. 96, 1989

Zhang, S-P. and Innanen, K. A.: 1988, Astron. J. 96, 1995 